\title{
Autosomal recessive or sex linked recessive: a counselling dilemma
}

\author{
I D YOUNG* , Z NUGENT $\dagger$, AND T GRIMM \\ From the Departments of Child Health* and Genetics $\dagger$, University of Leicester, Leicester; and $\ddagger$ the Institut \\ fur Humangenetik, Wurzburg, FRG.
}

SUMmARY This paper discusses the difficult problem that arises when information is sought by female relatives of two or more brothers, each of whom has an identical but undiagnosed or 'new' syndrome, which is likely to be either autosomal recessive or sex linked recessive in inheritance. It is proposed that standard Bayesian methods may be applied in this situation thus incorporating the prior probability for each event with conditional probabilities based upon pedigree analysis.

The purpose of this short paper is to draw attention to a problem which arises relatively frequently in clinical genetics, but which receives little attention in publications.

The problem to be considered is as follows. Information is sought by the healthy mother or sister of two or more males each of whom has an identical serious disorder, such as a malformation syndrome with mental retardation, which defies diagnosis. A careful history reveals that the parents are healthy and apparently unrelated, no-one else on either side of the extended family has a similar problem, and there is no evidence for a shared environmental insult.

In this situation it emerges that the mother has remarried, or is considering AID, while the sister wishes to start her own family. Each lady wishes to know her risk for having another similarly affected male infant.

Thus the essence of the problem is to derive from first principles the probability that the disorder in question is sex linked. Alternative genetic explanations include autosomal recessive inheritance, autosomal dominant inheritance with reduced penetrance, polygenic or multifactorial inheritance, and extranuclear inheritance, but in practice and for the purposes of this paper the crucial distinction rests between autosomal recessive and sex linked recessive inheritance.

\section{Approaches to the solution}

It is proposed that the problem is best approached

Received for publication 8 June 1985 .

Revised version accepted for publication 11 January 1985 by the application of conventional Bayesian analysis, which permits the relative probabilities for each pattern of inheritance to be determined. By this technique the products of the prior and conditional probabilities for each event, known as the joint probabilities, are compared to derive the posterior or relative probability for each.

THE PRIOR PROBABILITIES

Crucial to this exercise is the estimate of the prior probability ratio for autosomal recessive versus sex linked recessive inheritance. Three estimations are considered.

(1) Empiric estimate of the ratio of known autosomal recessive (AR) to sex linked recessive (XR) disorders. This can be readily obtained from a catalogue of all known human characteristics showing Mendelian inheritance, ${ }^{1}$ which yields an AR:XR ratio of approximately $6: 1$, or from a compilation of syndromes ${ }^{2}$ yielding a ratio of approximately 7.5:1.

This estimate is biased in favour of sex linkage on several counts. Firstly, the possibility still exists that in some societies males with a problem are more $N$ likely to come to medical attention than females. Secondly, it is unlikely that a 'new' autosomal recessive condition affecting only one member of a $\omega$ sibship of unrelated parents would be recognised as Mendelian, but the presence of another affected $\varrho$ male in the maternal line would alert the physician $\mathbb{D}$ to the possibility of sex linkage. Finally, since a recessive mutation on the $\mathrm{X}$ chromosome is much 70 more 'visible' than on an autosome, disorders

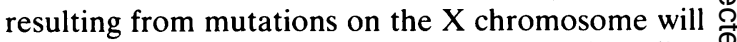
be preferentially ascertained in an outbreeding $\stackrel{\mathbb{Q}}{\square}$ society in which it may take many generations for an 
autosomal recessive mutation to 'find itself'. Thus, in the early editions of a catalogue of human Mendelian disease a relative excess of sex linked disorders would be anticipated. This bias is illustrated by comparing the AR:XR ratios for disorders known in mice, which are inbred in laboratory situations, with those known in man. The ratio in mice is approximately $14: 1{ }^{1}$ which compares closely with the autosome:X chromosome length in mice of 15:1.

(2) Theoretical estimate of the ratio of all potential autosomal recessive and sex linked recessive disorders. If it is assumed that there is an equal density of active loci on the autosomes and $\mathrm{X}$ chromosome, then this ratio is given by comparison of the length of all the autosomes with that of the $\mathrm{X}$ chromosome. In man the $\mathrm{X}$ chromosome constitutes approximately $5 \%$ of the haploid genome giving an AR:XR ratio of 19:1.

This ratio will be appropriate in years to come when considering a priori whether a particular recessively inherited disorder is more likely to be autosomal or sex linked. Whether this ratio is valid when considering an individual family is questionable.

(3) Theoretical estimate based on the relative probabilities of common parental heterozygosity (AR) versus maternal hemizygosity (XR). The point at issue here concerns the individual family and compares the relative probability that the parents will each be heterozygous for the same mutant allele at a particular locus (AR) with the probability that the mother will be a carrier at an $\mathrm{X}$ linked locus (XR).

For an autosomal recessive disorder which is in equilibrium, the prior probability that the parents will both be heterozygous for the same mutant allele at a particular locus

$$
\begin{aligned}
& =2 p q \times 2 p q(p+q=1) \\
& =4 p^{2} q^{2} \\
& =4 q^{2}(\text { since } p \text { is very close to } 1) \\
& =4 \mathrm{u}\left(\text { since } \mathrm{u}=\mathrm{q}^{2}(1-\mathrm{f})\right. \\
& \left.=\mathrm{q}^{2} \text { if } \mathrm{f}=0\right) .
\end{aligned}
$$

For a sex linked recessive disorder with fitness equal to zero, the prior probability that a mother will be a carrier at a particular $\mathrm{X}$ chromosome locus $=4 \mathrm{u}$.

Thus the prior probability ratio for $\mathrm{AR}$ :XR will be

$$
\frac{\frac{N a !}{(N a-1) !}\left(1-4 u_{a}\right)^{N a-1} 4 u_{a}}{\frac{N x !}{(N x-1) !}\left(1-4 u_{x}\right)^{N x-1} 4 u_{x}}
$$

where $\mathrm{Na}=$ number of autosomal loci in haploid genome

$\mathrm{Nx}=$ number of sex linked loci

$\mathrm{u}_{\mathrm{a}}=$ autosomal mutation rate

$\mathrm{u}_{\mathrm{x}}=$ sex chromosome mutation rate.

This ratio reduces to

$19(1-4 \mathrm{u}){ }^{(\mathrm{Na}-\mathrm{N} x)}$

if $u=u_{a}=u_{x}$ and $N a: N x=19: 1$.

Using this approach values for the AR:XR prior probability ratio are obtained as follows.

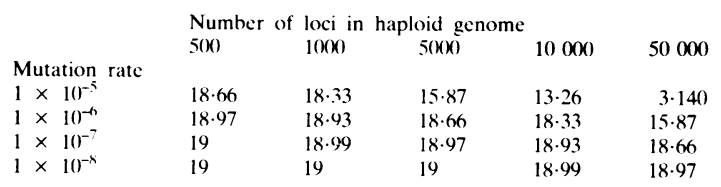

Since the hypothetical disorder in question is likely to be very rare with a mutation rate of at most $1 \times 10^{-6}$, it readily becomes apparent that regardless of the number of loci, the prior probability ratio approximates to 19:1.

Thus in effect estimates (2) and (3) both yield the same result, that is, an AR:XR prior probability ratio of 19:1.

THE CONDITIONAL PROBABILITIES

These include the structure of the sibship and the number of healthy males in the maternal line. This is illustrated in the worked example shown below in which the hypothetical sibship consists of two

\begin{tabular}{|c|c|c|c|}
\hline & \multicolumn{3}{|c|}{ Sex-linked recessive } \\
\hline & $\begin{array}{l}\text { Autosomal } \\
\text { recessive }\end{array}$ & $\begin{array}{l}\text { Mother = new } \\
\text { mutation }\end{array}$ & $\begin{array}{l}\text { Grandmother } \\
\text { a carrier }\end{array}$ \\
\hline \multicolumn{4}{|l|}{ Probability } \\
\hline Prior & $19 \cdot 0$ & 0.5 & 0.5 \\
\hline \multicolumn{4}{|l|}{ Conditional } \\
\hline (1) Two affected boys & $1 / 16$ & $1 / 4$ & $1 / 4$ \\
\hline (2) One healthy boy & $3 / 4$ & $1 / 2$ & $1 / 2$ \\
\hline (3) Two healthy girls & $9 / 16$ & 1 & 1 \\
\hline (4) Two healthy uncles & 1 & 1 & $1 / 4$ \\
\hline (5) Two healthy male & & & \\
\hline cousins & 1 & 1 & $\begin{array}{l}1 / 2+1 / 2(1 / 2)^{2} \\
=5 / 8\end{array}$ \\
\hline Joint & $(0.5010)$ & 0.11625 & 0.0098 \\
\hline
\end{tabular}
affected boys, one healthy boy, and two healthy girls. The mother has two healthy brothers and one healthy sister who in turn has two healthy sons. A prior probability ratio of 19:1 as derived previously is used.

In this calculation it is accepted that the proportion of new mutants among female heterozygotes is 0.5 , this being independent of the relative mutation rates in males and females, as derived elsewhere. ${ }^{3}$

Thus, in this example the posterior or relative probability of sex linkage equals $0 \cdot 126$ or approximately 1 in 8 . Thus, the mother of these boys could be informed that with a different spouse (or AID), the likelihood that her next child would be affected equals approximately 1 in 32 . 


\section{Discussion}

Attempts to address this problem in publications are few. In a description of two retarded dysmorphic brothers it was suggested that their disorder was more likely to be sex linked than autosomal, ${ }^{4}$ based on reference to an earlier paper in which a method for predicting the relative odds of autosomal recessive and sex linked recessive inheritance was developed and applied to defined groups of children with different mucopolysaccharidoses. ${ }^{5}$ Strictly speaking, these odds should only be applied to families within the study groups, and not to a family with a totally different disorder. When the disorder in question is lethal either in utero or in early infancy then the method presented by Harris et al can be considered. This is based on the probabilities of the maternal and grandmaternal sibships given different sex ratios, that is, $M: F=1: 1$ and $1: 2$.

While the provision of a risk estimate is only one aspect of genetic counselling, and it can be argued that the consultand is interested more in whether a risk is high or low than in actual figures calculated to the last decimal point, it is important that those involved in the provision of genetic information should be able to derive a reasonably accurate estimate of possible risks.

The situation discussed in this paper presents a particularly difficult exercise both in logic and in execution, and it is accepted that to a certain extent this problem is insoluble since several important assumptions are necessary. These include (1) an equal density of loci on the autosomes and sex chromosomes, (2) the attainment of equilibrium if the disorder is autosomal recessive, and (3) equal mutation rates on the autosomes and sex chromo- somes. All of these assumptions seem reasonable until proven otherwise. Consequently it is concluded that the approach as outlined herein is valid and it is hoped that the method presented here will prove helpful to those confronted by this difficult counselling situation.

The authors wish to thank their many colleagues who have joined in stimulating discussion of this problem, and in particular the anonymous referee whose helpful comments have been incorporated in this revised version. Special thanks also go to Mrs Susan Kenney for typing the manuscript. This paper was presented in preliminary form at the Clinical Genetics Society in November 1983.

\section{References}

1 McKusick VA. Mendelian inheritance in man. 6th ed. Baltimore: Johns Hopkins University Press, 1983.

${ }^{2}$ Smith DW. Recognizable patterns of human malformation. 3rd ed. Philadelphia: Saunders, 1982.

${ }^{3}$ Francke U, Felsenstein J, Gartler SM, et al. The occurrence of new mutants in the X-linked recessive Lesch-Nyhan disease. $A m$ J Hum Genet 1976;28:123-37.

${ }^{4}$ Smith RD, Fineman RM, Myers GG. Short stature, psychomotor retardation, and unusual facial appearance in two brothers. Am J Med Genet 1980;7:5-9.

5 Frota-Pessoa O, Opitz JM, Leroy JG, Patau K. Counselling in diseases produced either by autosomal or X-linked recessive mutations. Acta Genet 1968;18:521-33.

${ }^{6}$ Harris DJ, Ashcraft KW, Beatty EC, Holder TM, Leonidas JC Natal teeth, patent ductus arteriosus and intestinal pseudoobstruction: a lethal syndrome in the newborn. Clin Genet 1976;9:479-82.

Correspondence and requests for reprints to Dr I D Young, Department of Child Health, Clinical Sciences Building, Leicester Royal Infirmary, PO Box 65, Leicester LE2 7LX. 